\title{
Biobibliografía de los jesuitas de la estancia de Alta Gracia (Córdoba-Argentina).
}

\author{
Biobibliography of the Jesuits from the estancia of Alta Gracia \\ (Córdoba-Argentina).
}

Silvana M. Lovay*

Resumen: La contribución apunta a identificar y valorar las casi desconocidas figuras que a lo largo de 124 años formaron y desarrollaron la estancia jesuítica de Alta Gracia. Hoy transformada en una importante ciudad de la provincia de Córdoba, que conservó la antigua iglesia y edificio que los albergó. Aquí se mencionan las biografías de cada uno de los jesuitas que hemos podido localizar y que administraron o murieron en la estancia bajo distintas circunstancias. Igualmente constituyen solo una parte de la población estable que comenzó a surgir en 1643, con una amplia mayoría de africanos y afroamericanos esclavizados.

Palabras clave: Alta Gracia, estancias, Provincia jesuítica del Paraguay, estancieros.

\begin{abstract}
The contribution aims to identify and evaluate the almost unknown figures that throughout 124 years formed and developed the Jesuit estancia of Alta Gracia. Today transformed into an important city of the province of Cordova, that conserved the old church and building that hosted them. Here we mention the biographies of each of the Jesuits that we have been able to locate and who managed or died in the estancia under different circumstances. They also constitute only a part of the stable population that began to emerge in 1643, with a large majority of africans and African-american enslaved.
\end{abstract}

Key words: Alta Gracia, ranches, Jesuit Province of Paraguay, administrator

\footnotetext{
* Programa Antiguos jesuitas en Iberoamérica. CONICET-CIECS/UNC. E-mail: lovaysil@hotmail.com
} 
Recibido: 20 de junio de 2016

Evaluado: 15 de agosto de 2016 


\section{Introducción:}

Para la construcción de estas biografías nos valimos en primer lugar del libro de Page sobre Alta Gracia y del Libro de Cuentas de la estancia, donde pudimos conformar una primera lista ${ }^{1}$. Una vez obtenida, recurrimos al Catálogo del P. Storni, donde encontramos otras referencias y por supuesto, los datos más generales de los jesuitas que pasaron por Alta Gracia, que nos llevaron a obviar sus fuentes, tomadas sobre todo del ARSI. Por otra parte en las Cartas Anuas, que habitualmente insertan las notas necrológicas de los fallecidos en el período, encontramos escasa información en las de 16891700, 1714-1720 y 1750-1756 sobre el P. Matheo, el H. Vidal y los estudiantes Reyes y Santos, que mueren jóvenes. En la Sala IX del AGN encontramos cartas dirigidas o bien escritas por los PP. Beracierto, del Castillo, Molina, del Pino, Rauth y Requeta, en cuestiones referentes al desempeño de las estancias. Por otra parte el P. Grenón publicó 295 renuncias de bienes, donde figuran a su vez los datos de filiación familiar, que los primeros provinciales dejaron testimonio, aunque cayó en desuso en los catálogos trienales y secretos del siglo XVIII. De los aquí mencionados, el único que dejó sus bienes a la Compañía de Jesús fue Alonso Nieto de Herrera, exactamente la estancia de Alta Gracia. Personaje que nos ocupamos en otro lugar ${ }^{2}$. Por eso lo incluimos, aunque no tuviera intervención posterior en la estancia. El H. Nieto de Herrera tuvo un sobrino que vino de España y se sumó a la Compañía de Jesús, al igual que su primo Francisco Castañeda, ambos sacerdotes.

Diversos datos podemos extraer de los 26 jesuitas que hemos podido registrar su presencia en la estancia. De ellos, 10 eran sacerdotes, y excepto 2, todos profesos de cuatro votos. Coadjutores hubo 12 y estudiantes 3 . Estos últimos eran todos criollos y fallecieron jóvenes, entre 26 y 29 años. Solo de uno sabemos que fue por un accidente, llamado Ignacio de Reyes, del que brinda testimonio el P. Muriel.

En cuanto al origen, la mayoría son españoles, precisamente 14 de ellos; provenientes en su mayoría del País Vasco 5 y de Andalucía 3. Siguen 1 catalán, 1 madrileño, 1 de la comunidad de Castilla-León, 1 valenciano, 1 aragonés y 1 extremeño. Mientras luego siguen los criollos en número de 7, provenientes de Córdoba, Salta, Asunción, Buenos Aires y Catamarca. Concluyendo con 2 bávaros y 1 portugués.

Si no contamos a los jóvenes estudiantes fallecidos por accidente, la edad promedio de expectativa de vida es de entre 62 y 63 años, con un mínimo de 28 del P. Cortés del que no conocemos la causa de su deceso y un máximo de 86 para el H. Vidal y 87 para el H. Nieto de Herrera. De ellos están sepultados en la iglesia (o estuvieron antes de la reciente remoción de cadáveres) seis: los PP. Aparicio y Cortés, los estudiantes Cáceres, Reyes y Santos, además de Torres del que no pudimos identificar sus estado eclesiástico. Al momento de la expulsión se encontraban los PP. López y Molina, junto el H. Benito que morirán en el exilio. El último de todos fue el P. López en 1779.

La estancia recibió muchas visitas a lo largo de su historia, sobre todo de los provinciales, que dejaron su testimonio en el Libro de Cuentas y en los Memoriales ${ }^{3}$.

\footnotetext{
${ }^{1}$ Obviamos tratar el tema de los hermanos estancieros y de los reglamentos para la administración de estancias ampliamente trabajados en Page 2004 y 2008.

${ }^{2}$ Lovay, 2013.

${ }^{3}$ En el Libro de Cuentas quedaron asentadas las visitas de los PP. Juan Bautista Zea (1718), quien es el que ordena comenzar el libro, José de Aguirre (1720 y 1721), Luis de la Roca (1723), Ignacio de Arteaga (1726), Jerónimo Herrán (1733), Jaime Aguilar (1734 y 1736), Antonio Machoni (1741), Bernardo Nusdorffer (1745), Manuel Querini (1747 y 1750), José de Barreda (1753 y 1754) y Nicolás Contucci (1760).
} 
Aunque de los cinco conocidos e impersonales; solo el provincial Querini menciona al H. Raith $(1747)^{4}$. Pero también pasaron por sus claustros diversos misioneros, profesores y alumnos que no dejaron registro. De estos se destacan tres personajes verdaderamente emblemáticos como Juan de Escandón (1696-1772), Domingo Muriel (17181795) y Florián Paucke (1719-1779), que incluso dejaron escrito su paso por la estancia $^{5}$.

Ningún biógrafo contemporáneo a los jesuitas de Alta Gracia se ocupó de alguno de ellos. Ni Orosz o Peramás, entre los más conocidos y prolíferos trabajadores del género. Tampoco figuran en el Diccionario Histórico Geográfico de la Compañía de Jesús, y el P. Gracia solo menciona a Carbalho y a Klausner, este último referenciado por Sierra y varios otros a partir de sus cartas, publicadas primeramente por el P. Mühn en la Revista del Instituto Histórico y Geográfico de Montevideo en 1930 y luego de más de una década las completó y publicó en un libro. Igual pasa con el P. Furlong, quien solo menciona a los que fueron misioneros entre guaraníes.

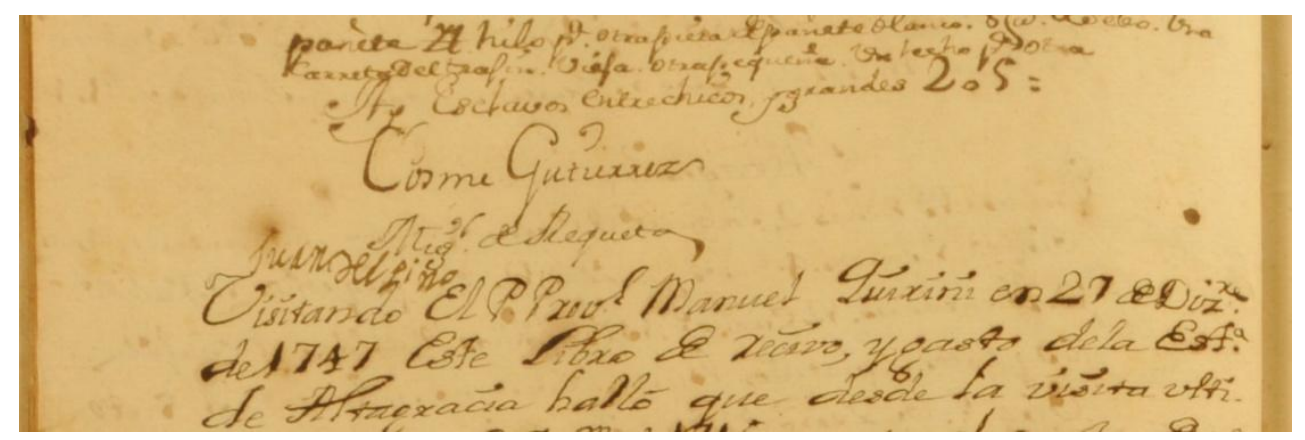

Firmas de los HH. Cosme Gutiérrez, Miguel Requeta y Juan del Pino en la visita que hizo a la estancia el provincial P. Manuel Querini en 1747.

\section{Noticias biográficas}

Aparicio, Benigno Eugenio: Nació en Madrid el 20-11-1676, ingresando a la Provincia del Paraguay el 15-10-1694. Llegó a Buenos Aires el 24-9-1698 en la expedición del P. Ignacio de Frías. Recibió el sacerdocio del obispo agustino de origen peruano fray Martín de Híjar y Mendoza el 6-4-1704, cuando dejaba la sede de Concepción (Chile). Profesó su cuarto voto en Tarija el 15-8-1712. Falleció en Alta Gracia el 14-6-1741 ${ }^{6}$.

Bailina, Simón: Nació en Berga, Barcelona, el 8-12-1693, ingresando a la Provincia del Paraguay el 4-5-1716. Llegó a Buenos Aires el 13-7-1717 en la expedicón de los PP. Bartolomé Jiménez y José de Aguirre. Administró la estancia entre 1722 y 1723, recibiéndola del P. del Pino con 156 esclavos. Profesó su cuarto voto en Córdoba el 15-81729. Fue rector en el colegio de Santiago del Estero en 1737, luego designado procurador a Europa electo en 1744, junto con el P. Orosz y Morales, quien falleció en Madrid, mientras que Bailina no viajó. Sí lo hizo cuando fue designado en la Congregación del 31-10-1757, junto con los PP. Juan de Escandón y Antonio Gutiérrez. Este último

Los Memoriales a la estancia están firmados por Luis de la Roca (1715, 1723), Jaime de Aguilar (1734), Antonio Machoni (1741), Bernardo Nusdorffer (1745) y Manuel Querini (1747).

${ }^{4}$ Barrabino, 2013: 229.

5 Page, 2000: 54-58.

${ }^{6}$ Storni, 1980: 16. Page, 2007: 46. 
no viajó, y el ya anciano P. Bailina falleció en Madrid el 1-4-1760. Solo regresó el P. Escandón cuatro años después ${ }^{7}$.

Benito, Francisco: Nació en Segovia el 15-5-1749, ingresando a la Provincia del Paraguay el 12-7-1745. Llegó a Buenos Aires tres días después en la expedición de los PP. Diego Garvia y Juan José Rico, profesando sus primeros votos el 12-7-1747 y sus últimos como coadjutor en el colegio de Belén el 2-2-1757. Para la expulsión se encontraba en Alta Gracia. Falleció en Roma el 9-6-1779 ${ }^{8}$.

B[V]eracierto, Juan Bautista: Nació en Deva, Guipúzcoa el 25-5-1700, ingresando a la Provincia del Paraguay el 9-10-1721. Recibió la estancia en 1733 de manos del H. Klausner, siendo anteriormente administrador de estancia La Candelaria. Desde ella escribe una carta (29-2-1728) al mismo H. Klausner sobre la huida de un conchabado. También se conservan cartas que le dirigieron el P. Miguel López, el H. Francisco Mareca sobre cuestiones de ganados, P. Diego Garvia y del H. Echezárraga varias cartas sobre trabajo de peones, comercio de ganados, yerba y vino, además de otras cuestiones. Fue trasladado de Candelaria y reemplazado por el H. Antonio Muñoz el 17-11-1728. Sus últimos votos como coadjutor los profesó en Córdoba el 6-1-1734. Falleció en Santiago del Estero el 23-1-17409.

Cáceres, Ramón de: Nació en Asunción el 22-4-1684, ingresando a la Provincia del Paraguay el 13-5-1706 y falleciendo como estudiante en Alta Gracia en $1713^{10}$.

Carbalho, Benito: Nació en Oporto el 20-3-1619, ingresando a la Provincia del Paraguay el 11-11-1647. Fue mencionado como administrador en un documento del año 1653. Profesó sus últimos votos como coadjutor en Córdoba el 31-7-1660. Lo último que se sabe de él es que estaba en Buenos Aires para $1674^{11}$.

Castillo, Antonio del: Nació en Durango, Vizcaya el 29-10-1702, ingresando a la Provincia del Paraguay el 19-12-1725. Profesó sus últimos votos como coadjutor en Salta el 9-6-1737. Administrador de la estancia la entrega a fines de 1753 al P. Juan Rojas. Para la expulsión se encontraba en el colegio de Córdoba, falleciendo en su exilio de Faenza el 22-4-1774 ${ }^{12}$.

Cortés, Antonio: Hijo de Juan Antonio y de Isabel Benedito, nació en Gerice, Valencia el 25-8-1714, ingresando a la Provincia del Paraguay el 31-10-1733. Previamente hizo renuncia de bienes a favor de su madre y a su muerte de sus hermanos José y Ana María por partes iguales. Llegó a Buenos Aires el 25-3-1734 en la expedición de los PP. Antonio Machoni y Sebastián de San Martín. Profesó sus primeros votos el 1-11-1735, obteniendo el sacerdocio el 4-11-1740. Falleció en Alta Gracia el 14-1-1742 ${ }^{13}$.

Ellacurriaga, Pedro. Hijo de Juan y de Catalina de Dum, nació en Durango, Vizcaya, el 21-1-1706, ingresando a la Provincia del Paraguay el 12-7-1728. Llegó a Buenos Aires el 19-4-1729 en la expedición de los PP. Jerónimo Herrán y Juan de Alzoa. Sus primeros

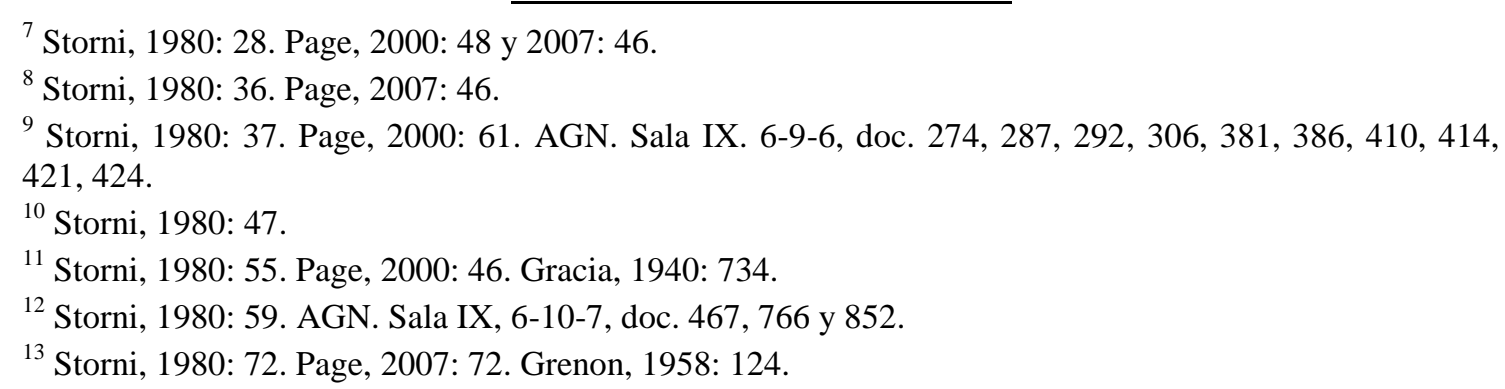


votos los obtuvo el13-7-1730 y los últimos como coadjutor en Asunción el 26-7-1739. Hizo renuncia de bienes a favor de su primo hermano del mismo nombre en 1732. Para la expulsión se encontraba en el colegio de San Ignacio de Buenos Aires (3-7-1767), falleciendo en el exilio en Génova en marzo de $1779^{14}$.

Gutiérrez, Cosme: Nació en Cádiz el 4-2-1704, ingresando a la Provincia del Paraguay el 18-5-1739. Sus primeros votos los profesó el 19-5-1741 y sus últimos como coadjutor en Asunción el 1-3-1750. Su firma se encuentra en el Libro de Cuentas de la estancia en 1747 junto a la de Miguel Requeta y Juan del Pino. La expulsión lo encuentra en el colegio de Asunción (30-7-1767) y muere en Faenza el 28-12-1776 ${ }^{15}$.

K[C]lausner, José: Nació en Kammlach, Baviera el 15-2-1685, ingresando a la Provincia del Paraguay el 23-7-1717. Llegó a Buenos Aires el 13-7-1717 en la expedición de los PP. Bartolomé Jiménez y José de Aguirre. Profesó sus últimos votos como coadjutor el 3-12-1727. Fue administrador de la estancia entre 1723 y 1731. Tiempo en que fue visitada por el obispo del Tucumán José Antonio Gutiérrez de Zevallos. Peltrero de oficio, hizo construir la herrería de la estancia. La misma contaba para cuando la entregó con unos 200 habitantes esclavizados. Según su propio testimonio además fundía campanas, fabricaba lámparas, hacía de barbero y tonelero, e instruía a los indios en el oficio, habiendo pasado también por las estancias de Santa Catalina, Jesús María, Caroya, la de San Miguel y procurador en del colegio de La Rioja. Trasladado al Convictorio de Monserrat, creó allí una pequeña fábrica de utensilios de peltre, falleciendo allí el 20-5$1746^{16}$.

López, Pedro Nolasco: Hijo de Tomás López y Fernández y de Catalina de Córdoba, nació en Córdoba, Argentina, el 30-1-1739, ingresando a la Provincia del Paraguay el 20-11-1757. Hizo renuncia de bienes en 1763 a favor de su hermana María Rosa. Para la expulsión se encontraba en Alta Gracia (12-7-1767). En su exilio en Italia profesó su cuarto voto el 2-2-1772 en Faenza, donde muere el 1-10-1799 ${ }^{17}$.

Matheo, José: Nació en Jabaloyas, Teruel, el 1-9-1670, ingresando a la Provincia del Paraguay el 9-4-1696. Llegó a Buenos Aires el 24-4-1698 en la expedición de Ignacio de Frías. Fue administrador, antecesor del P. Carbalho. Profesó su cuarto voto en Córdoba el 15-8-1711, falleciendo en Salta el 5-4-1718. Su noticia necrológica aparece en la Carta Anua de manera muy sintética, mencionando que murió de una gravísima y muy molesta enfermedad ${ }^{18}$.

Molina, Juan de: Hijo de Francisco y Juana de Salas, nació en Catamarca el 4-5-1734, ingresando a la Provincia del Paraguay el 15-9-1754. Obtuvo el sacerdocio en 1759 y al año siguiente hizo renuncia de bienes a favor de su madre. El 19-5-1761 le escribe al P. visitador rogándole le envíe a las reducciones. Fue cura en la reducción de Santo Tomás entre 1742 y 1751, luego de Trinidad hacia 1755 y de Yapeyú. Para la expulsión se encontraba en Alta Gracia (12-7-1767). En el exilio profesó su cuarto voto en Imola el 263-1769, falleciendo en Ravena el 4-7-1778 ${ }^{19}$.

\footnotetext{
${ }^{14}$ Storni, 1980: 89. Page, 2007: 46. Grenón, 1958: 123.

${ }^{15}$ Storni, 1980: 133-134. LC II: 214.

${ }^{16}$ Storni, 1980: 153. Page, 2000: 53-54 y 2007: 46. Gracia, 1940: 393. Sierra, 1944: 395.

${ }^{17}$ Storni, 1980: 166. Grenón, 1958: 131.

${ }^{18}$ Storni, 1980: 180. Page, 2007: 46. CA 1714-1720, f. 359v.

${ }^{19}$ Storni, 1980: 188. Grenón, 1958: 130. Furlong, 1963: 139, 155 y 157. AGN. Sala IX, 6-10-5, doc. 133.
} 
Nieto de Herrera, Alonso: Hijo de Francisco Nieto y Catalina Medina, nació en Garrovillas de Alconétar, Cáceres en 1574. De España pasó al Perú, donde la Audiencia de La Plata le otorgó el real título de escribano. Al quedar viudo y por consejo de su sobrino Francisco Nieto (obituario en Carta Anua 1663-1666), ambos ingresaron a la Provincia del Paraguay el 24-6-1643. En tanto que el escribano hizo renuncia de la estancia de Alta Gracia a favor de la Compañía de Jesús. Profesó sus últimos votos como coadjutor el 31-7-1655, falleciendo en Córdoba en 1661. Su obituario en la Carta Anua 1659$1662^{20}$.

Pino, Juan Antonio del: Nació en Montilla el 27-10-1690, ingresando a la Provincia del Paraguay el 15-5-1710. Llegó a Buenos Aires el 8-4-1712 en la expedición del P Francisco Burgés. Profesó sus últimos votos como coadjutor el 5-10-1721 y al año siguiente entregó la administración de la estancia al H. Bailina, falleciendo en la ciudad de Córdoba el 9-5-1763. En 1722 entregó la estancia al H. Bailina, pero lo vemos de nuevo en 1743 por una carta que le escribe el capellán Pedro Pablo Canzinos y otra de Juan Sánchez Rodríguez. En 1747 se la entrega el H. Requedo por una ausencia temporal y la toma de nuevo para entregarla al H. Raith en $1748^{21}$.

Quintero, Bartolomé: Nació en Buenos Aires el 24-8-1635, ingresando a la Provincia del Paraguay el 7-9-1652. Se brinda referencias de su estadía en Alta Gracia en un documento de 1675 en que recibe las tierras vacas de los alrededores de la estancia. Fue por un tiempo procurador de la Provincia por ausencia del P. Calatayud. Profesó sus últimos votos el 2-2-1669 en Santiago del Estero, donde falleció el 2-1-1712 22 .

Raith, Jorge. Nació en Pfreimd, Baviera, el 12-6-1718, ingresando a la Compañía de Jesús de Alemania Superior el 17-9-1740. Llegó a Buenos Aires el 15-7-1745 de los PP. Diego Garvia y Juan José Rico. Administró la estancia recibiéndola del H. del Pino en 1748 y entregándola al H. Castillo. Profesó sus últimos como coadjutor en Córdoba el 2-2-1751. Se lo menciona también como panadero y conocemos varias cartas que lo mencionan entre 1763 y 1764 sobre el comercio de ganado, traslado de indios enfermos, actividades comerciales y el envío de ladrillos que le hizo Prudencio Ramírez. La expulsión lo encontró en el Colegio de Belén (3-7-1767). Regresó a su patria y falleció en Mindelheim, Baviera, el 4-6-1776 ${ }^{23}$.

Requeta, Miguel: Nació en Durango, Vizcaya el 17-2-1709, ingresando a la Provincia del Paraguay el 17-11-1728. Llegó a Buenos Aires el 19-4-1729 en la expedición de los PP. Jerónimo Herrán y Juan de Alzoa. Hizo renuncia de bienes en 1732. En 1738 el P. Joaquín de Yegros le otorgó un poder general para que lo represente en pleitos y negocios. Sus últimos votos como coadjutor los profesó en Santiago del Estero el 23-8-1739. Fue administrador, entregando la estancia al H. del Pino en 1747. Falleció en Tarija en $1766^{24}$.

Reyes, Ignacio de: Hijo de Francisco y de María Montoya, nació en Salta el 1-2-1726, ingresando a la Provincia del Paraguay el 8-1-1746, profesando sus primeros votos el 91-1748. Hizo renuncia de bienes en 1751 a favor de su madre. Falleció como estudiante en Alta Gracia el 23-1-1752. Su obituario en la Carta Anua de 1750-1756, aunque con

\footnotetext{
${ }^{20}$ Storni, 1980: 199. Grenón, 1955: 409. Lovay, 2013: 322-328.

${ }^{21}$ Storni, 1980: 222. Page, 2007: 46. AGN, Sala IX, 6-9-7, doc. 474 y 476.

${ }^{22}$ Storni, 1980: 231. Page, 2000: 46. Gracia, 1940: 348.

${ }^{23}$ Storni, 1980: 232. Page, 2000: 64. Sierra, 1944: 399. AGN, Sala IX, 6-10-6, doc. 180, 256 y 526.

${ }^{24}$ Storni, 1980: 236. Page, 2007: 46. Grenón, 1958: 123. AGN, Sala IX, 6-9-7, doc. 184.
} 
solo datos generales. Es mencionado por el P. Muriel al describir la estancia como un "joven de mucha virtud", que solía ensillar los caballos para sus paseos. Mientras hacía este trabajo una patada de un equino acabó con su vida ${ }^{25}$.

Rojas, Juan de: Nació en Asunción el 27-12-1718, ingresando a la Provincia del Paraguay el 19-1-1742. Profesó sus primeros votos el 20-1-1744, obteniendo el sacerdocio el 10-10-1752 y su cuarto voto el 15-8-1759. Para la expulsión se encontraba en Santa Fe (13-7-1767). Exiliado en Italia, muere en Faenza el 23-4-1794 ${ }^{26}$.

Ruiz de Llanos, Diego: Nació en Salta el 10-12-1670, ingresando a la Provincia del Paraguay el 12-6-1687. Profesó sus primeros votos el 13-6-1689 y el de cuarto voto en San Miguel de Tucumán el 14-9-1704. En 1716 fue nombrado rector del colegio de San Miguel de Tucumán. Desde entonces o poco después pasó a Córdoba, donde sucedió al P. Zavala en la administración de Alta Gracia, entre 1718 y 1722. Falleció en la reducción de Santa Ana el 11-5-1749 27 .

Santos, Domingo: Nació en Salta el 3-8-1729, ingresando a la provincia del Paraguay el 2-9-1748. Profesó sus primeros votos el 3-9-1750 y falleció siendo estudiante en Alta Gracia el 12-7-1755. Su obituario en la Carta Anua de 1750-1756, aunque con solo datos generales ${ }^{28}$.

Torres, Juan Luis: Solo sabemos que murió en Alta Gracia el 7-5-1763 ${ }^{29}$.

Vidal, Diego: Nació en Lebrija, Sevilla, en 1613, ingresando a la Provincia de Andalucía el 11-6-1633. Llegó a Buenos Aires el 20-12-1636 en la expedición del P. Juan Bautista Ferrufino. Profesó sus últimos votos como coadjutor en Córdoba el 11-2-1646, falleciendo a los 86 años en la reducción de Encarnación de Itapúa el 26-5-1699. En su obituario se menciona que también estuvo en las estancias de Jesús María de Córdoba, la de San Ignacio de Silipica de Santiago del Estero, la de los Lules en Tucumán, y la de San Lorenzo en Paraguay de donde pasó a las reducciones por su avanzada edad ${ }^{30}$.

Zavala, Lucas: Nació en Lequeito, Vizcaya, el 25-11-1682, ingresando a la Provincia del Paraguay el 9-10-1698. Profesó sus primeros votos el 1-12-1700 y el cuarto voto el 2-2-1717. Al año siguiente entrega la administración de la estancia al P. Diego Ruiz de Llanos. Fue rector del colegio de Asunción hacia 1737 y posteriormente elegido procurador a Europa el 20-11-1740, junto con los PP. Jaime Aguilar y Pedro Arroyo, pero la expedición no se concretó. Falleció en Asunción el 10-1-1743 ${ }^{31}$.

\section{Archivos citados}

AGN. Archivo General de la Nación Argentina. Sala IX.

CA. Carta Anua 1714-1720 (Traducción Leonhardt). Biblioteca Colegio del Salvador, Buenos Aires, Estante 12.

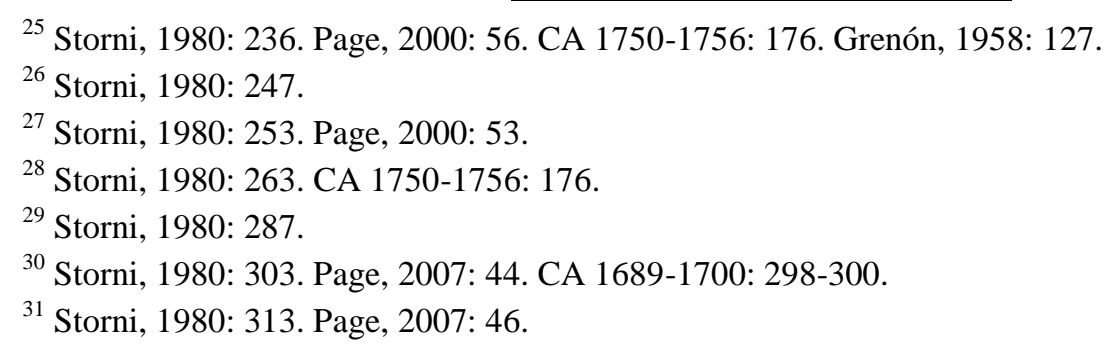


CA. Carta Anua 1750-1756 (Traducción Leonhardt). Biblioteca Colegio del Salvador, Buenos Aires, Estante 11.

LC. Libro de Cuentas de Alta Gracia. Lo que la estancia remite al Colegio y lo que en ella se gasta 1718-1767. Museo Casa del Virrey Liniers y Estancia Jesuítica de Alta Gracia.

\section{Bibliografía}

Barrabino, Martín (2013). "Memoriales de los Provinciales para los domicilios de Córdoba (2da parte)". IHS. Antiguos Jesuitas en Iberoamérica. CONICETCIECS/UNC, Vol. 2, No 2 .

Cartas Anuas de la Provincia Jesuítica del Paraguay 1681-1692. 1689-1692. 16891700 (2015). Introducción María Laura Salinas, Colaboración Julio Folkenand. Asunción: Centro de Estudios Antropológicos de la Universidad Católica

Furlong SJ, Guillermo (1962). Misiones y sus pueblos de guaraníes. Buenos Aires: Imprenta Balmes.

Grenón SJ, Pedro (1955). "Las renuncias de bienes en la provincia del Paraguay. Siglo XVII". Archivum Historicum Societatis Iesu. Roma. № 24.

- (1958). "Las renuncias de bienes en la provincia del Paraguay. Siglo XVIII". Archivum Historicum Societatis Iesu. Roma. № 27.

Lovay, Silvana M. (2013). "El ingreso de Francisco y Alonso Nieto de Herrera a la Compañía de Jesús". IHS. Antiguos jesuitas en Iberoamérica. Vol. 1 No1. Córdoba. CONICET-CIECS/UNC.

Mühn SJ, Juan (1946). El Río de la Plata visto por viajeros alemanes del siglo XVIII. Buenos Aires: Huarpes.

Page, Carlos A. (2000). La estancia jesuítica de Alta Gracia. Córdoba: Universidad Nacional y Católica de Córdoba.

--- (2004). "Los hermanos estancieros de la Compañía de Jesús y su actuación en la antigua provincia del Paraguay". Investigaciones y Ensayos. № 24. Academia Nacional de la Historia. Buenos Aires.

-- (2007). Los viajes de Europa a Buenos Aires según las crónicas de los jesuitas de los siglos XVII y XVIII. Córdoba: Báez ediciones.

-- (2008). "Reglamentos para el funcionamiento de las haciendas jesuíticas en la antigua provincia del Paraguay". Dieciocho, Hispanic Enlightenment, University of Virginia, US, $\mathrm{N}^{\mathrm{o}} 31.2$, otoño.

Sierra, Vicente D. (1944). Los jesuitas germanos en la conquista espiritual de HispanoAmérica. Siglos XVII-XVIII. Buenos Aires: Facultad de Filosofía y Teología / Institución Cultural Argentino-Germana.

Storni SJ, Hugo (1980). Catálogo de los jesuitas de la Provincia del Paraguay (Cuenca del Plata) 1585-1768). Roma: Institutum Historicum S.I. 